\title{
A Case Report: Internal Carotid Artery Dissection Presenting as Hoarseness Secondary to Vocal Cord Palsy
}

\author{
Authors: \\ *Foon Ng Kee Kwong, ${ }^{1}$ Fiona Brodie, ${ }^{2}$ Claire McArthur, ${ }^{3}$ Fiona \\ MacGregor ${ }^{4}$ \\ 1. ENT Department, Queen Elizabeth University Hospital, Glasgow, Scotland \\ 2. Care of the Elderly/Stroke Medicine, Coathill Hospital, Coatbridge, Scotland \\ 3. Radiology Department, Glasgow Royal Infirmary, Scotland \\ 4. ENT Department, Gartnavel General Hospital, Glasgow, Scotland \\ *Correspondence to foon.ngkeekwong@nhs.net
}

Disclosure: The authors have declared no conflicts of interest.

Received: $\quad 09.08 .20$

Accepted: $\quad 27.10 .20$

Keywords: Carotid, cord, dissection, hoarseness, palsy, vocal.

Citation:

EMJ Neurol. 2021;9[1]:66-71.

\section{Abstract}

Internal carotid artery (ICA) dissection is a rare cause of a cranial nerve $X$ palsy. Patients more commonly present with stroke or transient ischaemic attacks. An undetected and untreated ICA dissection can have serious consequences. Here, the authors present two cases of ICA dissection presenting with isolated vagal nerve palsy presenting with hoarseness. CT scans provided good evidence of ICA in both cases. The patients were treated with antiplatelet agents and made a good recovery with complete resolution of symptoms.

\section{INTRODUCTION}

Vocal cord paralysis or weakness can be a symptom of malignancy, caused by viral infection, iatrogenic, a result of trauma, idiopathic, or a result of brainstem infarction, in particular lateral medullary syndrome.' Internal carotid artery (ICA) dissection is a rare cause of an $\mathrm{X}$ cranial nerve palsy. This particular nerve palsy is present in only $16 \%$ of ICA dissection cases. ${ }^{2}$ Isolated cranial nerve palsies are an unusual presentation of ICA dissection. Patients more commonly present with stroke or transient ischaemic attacks. They can also present with multiple lower cranial nerve palsies and other concurrent neurological deficits, such as Horner's syndrome, due to compression of sympathetic nerves around the ICA by a

thrombus. ${ }^{2}$ An undetected and untreated ICA dissection can have serious consequences. ${ }^{3}$ Here, the authors present two cases of ICA dissection with isolated vagal nerve palsy.

\section{CASE 1}

\section{Presentation}

A previously healthy male, who occasionally smoked, presented at the age of 42 with a sudden onset of hoarseness and swallowing difficulties, but with no history of trauma. They also reported pain over the right side of their head and face. They were initially treated in primary care with antibiotics, but there was no improvement. A contrast-enhanced CT scan of 
their chest and abdomen did not reveal the cause of their symptoms. They were then referred to the otolaryngology service where a right vocal cord palsy was found on fibreoptic laryngoscopy. Due to some concern about the appearance of the right piriform fossa, they underwent examination under general anaesthetic, but this was unremarkable.

\section{Investigation}

A further CT scan was requested in order to investigate a potential cause of the vocal cord palsy in the head and neck. The standard protocol for contrast CT of the neck alone for otorhinolaryngological (ear, nose, and throat) pathology in the authors' local department was utilised. This is a split bolus technique: $45 \mathrm{~mL}$ of Omipaque ${ }^{\mathrm{TM}}$ (GE Healthcare, Chicago, Illinois, USA; iohexol) 300 administered at a rate of $1.5 \mathrm{~mL} / \mathrm{sec}$, with a further $45 \mathrm{~mL}$ bolus at the same rate with an inter-bolus delay of 30 secs. This resulted in neck images being acquired with good soft tissue and vascular contrast at 90 secs following the start of the first contrast injection. Axial coverage was from above the frontal sinuses to the clavicles. This CT scan demonstrated a thickening of the right ICA at the skull base with narrowing of the vessel lumen and eccentric hypodensity, strongly suggestive of arterial dissection (Figure 1). An MRI was also performed using axial fat-saturated sequences and this revealed corresponding eccentric T2 iso- or hyperintensity around the right ICA true lumen, confirming the suspicion of dissection (Figure 2).

\section{Treatment and Outcome}

Antiplatelet therapy was commenced for a period of 6 months. Over the next 6 months, their vocal cord palsy persisted but their left vocal cord was compensating very well, allowing them to produce a stable and strong voice. They were referred to the speech and language therapy team, and a year after their first presentation their right vocal cord had fully recovered.

\section{CASE 2}

\section{Presentation}

A 29-year-old female patient, previously healthy, presented with a sudden onset of difficulty swallowing. Shortly after the onset of their dysphagia, they developed hoarseness. Although their physical activities were not affected, they found themselves more breathless walking up the stairs and found it difficult to complete sentences. They also noticed a left-sided pulsatile tinnitus when lying on their left side. They attended their general practitioner and were treated with two courses of antibiotics. They reported no history of trauma. Onward referral was made to the otolaryngology service. Further investigation was arranged due to the unresolved cause of the issue. Fibreoptic examination revealed impaired left vocal cord movement.

\section{Investigation}

A CT scan was organised, and the split-bolus contrast CT showed narrowing of the lumen of the left ICA and eccentric mural thickening, consistent with a left ICA dissection (Figure 3). The same split-bolus sequence was used. A 75 $\mathrm{mL}$ bolus was administered at $2.5 \mathrm{~mL} / \mathrm{sec}$ and the thorax was scanned at 65 secs delay with arms up. This was quickly followed by a $50 \mathrm{~mL}$ bolus at $2.0 \mathrm{~mL} / \mathrm{sec}$ and the neck was scanned with arms down at 25 secs delay. The result was a split-bolus neck scan at approximately 100 secs following the start of the first injection.

\section{Treatment and Outcome}

Following the diagnosis, the individual was referred to the stroke team and began antiplatelet therapy for a duration of 6 months to reduce the risk of thromboembolism. ${ }^{4}$ Followup CT angiogram revealed a near-complete resolution. When reviewed a few months later, the patient reported that their swallowing and voice symptoms had completely resolved. The only residual symptom was the awareness of intermittent pulsatile tinnitus in their left ear at night. Genetic testing was carried out and no underlying genetic connective tissue disorder was discovered.

\section{DISCUSSION}

ICA dissection can be either intracranial or extracranial. Although rare with an incidence between 2.6 and 2.9 per 100,000, extracranial ICA dissection is still more common than intracranial ICA in the adult European population. ${ }^{5}$ It is also more common in males, usually aged 50-59. ${ }^{6}$ 


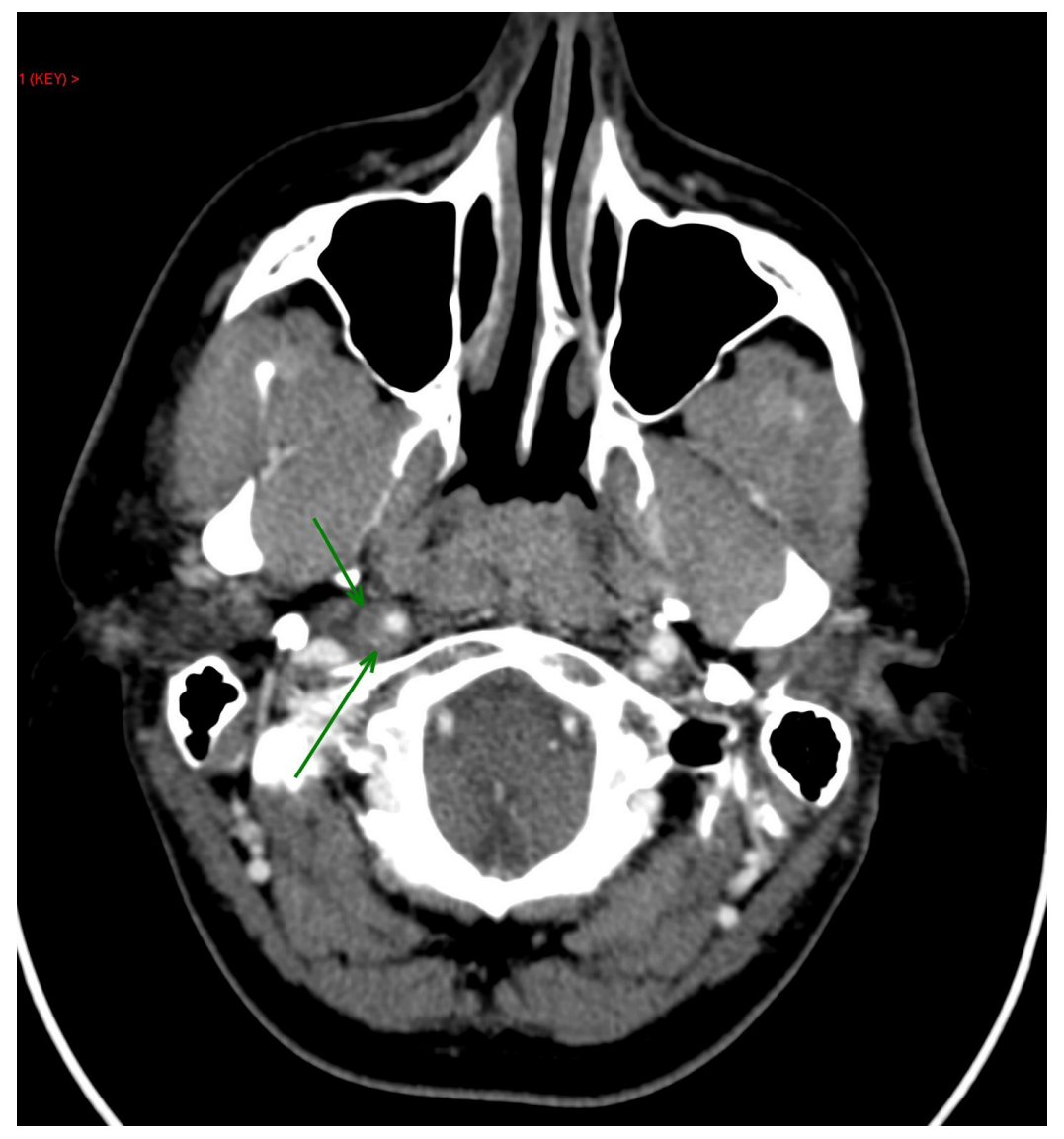

Figure 1: Axial CT image depicting the intramural haematoma.

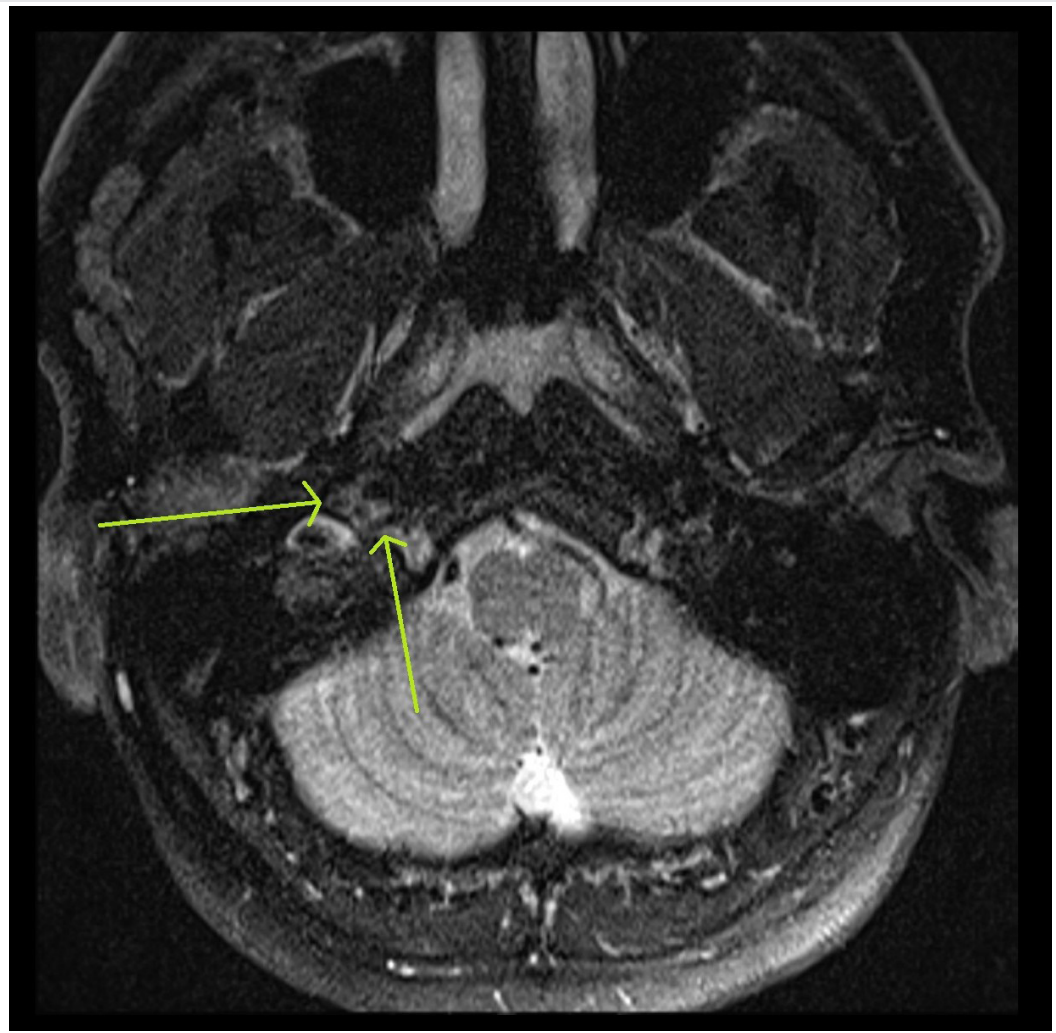

Figure 2: Axial MRI image of the intramural haematoma. 


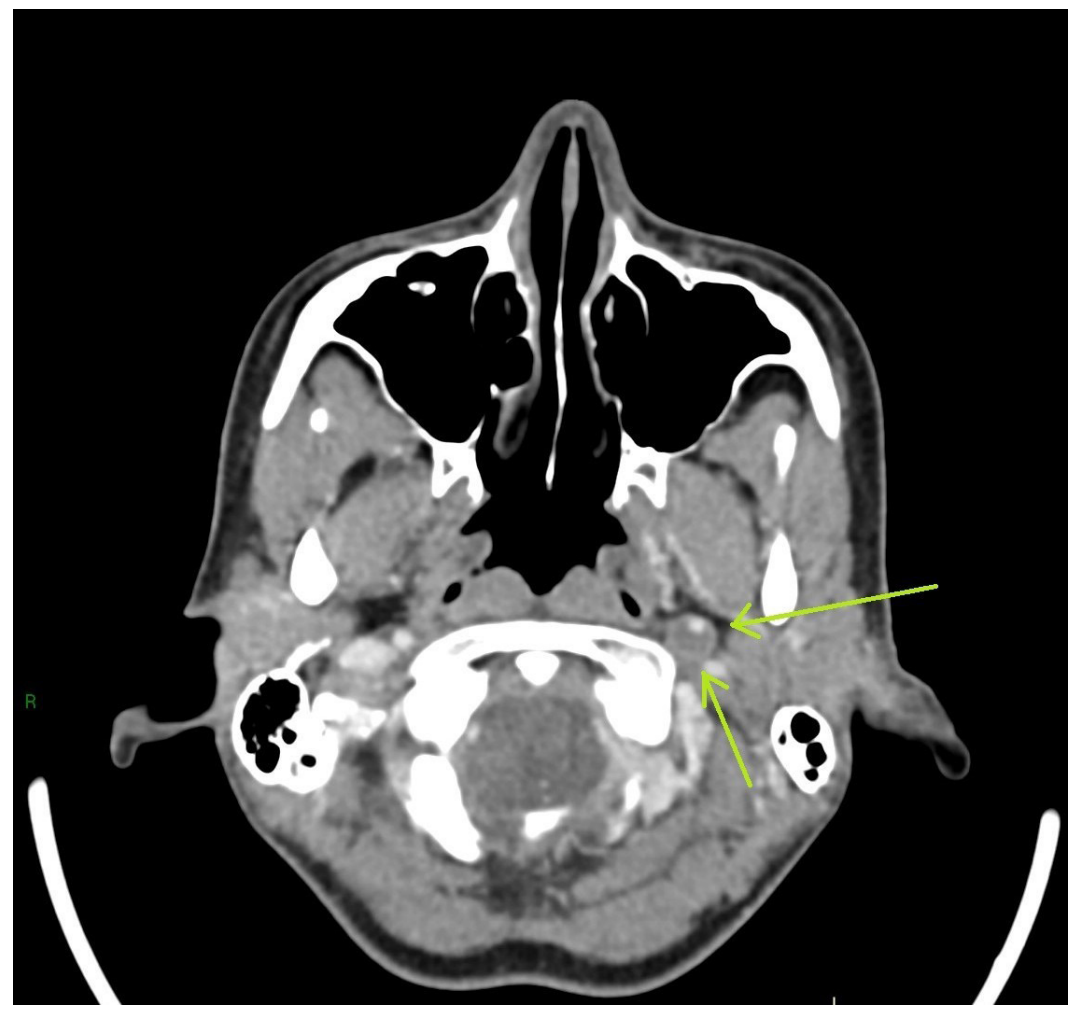

Figure 3: Axial CT image depicting the intramural haematoma.

It presents with stroke in $67 \%$ of cases and Horner's syndrome in $28-58 \%$ of cases. ${ }^{7}$ Other symptoms include ipsilateral neck, facial or eye pain, ptosis, and visual disturbance., ${ }^{1,3,8}$ Approximately $3-16 \%$ of patients with ICA dissection present with lower cranial nerve palsies. The most affected cranial nerves are $\mathrm{X}$, $\mathrm{XI}$, and XII. 3,8,9 As they exit the skull base through the jugular foramen and hypoglossal canal, these nerves travel in the carotid sheath and are therefore in close relation to the ICA, especially cranial nerves $X$ and $X I I$, which travel a longer distance along its length. In the neck, the vagus nerve can be found on both sides, and enters the carotid sheath and travels inferiorly between the common carotid artery and the internal jugular vein all the way to the base of the neck. The left vagus nerve then branches and gives rise to the left recurrent laryngeal nerve, which loops around the aortic arch, while the right vagus nerve branches at the level of the right subclavian artery to loop around it. The recurrent laryngeal nerves travel back up to provide motor innervations to the vocal cords. The vagus nerve also contributes to the oesophageal plexus by sending parasympathetic and afferent fibres.
Mass effect from an ICA haematoma on the vagus nerve can give rise to hoarseness or dysphagia or both. It is interesting to note that ICA dissections that exhibit lower cranial nerve paralysis are less often associated with cerebral ischaemia. ${ }^{3}$ The cervical plexus lies deep to the carotid sheath. It provides sensory innervations to the ear, scalp, neck, and upper part of the chest. Compression of these nerves could account for the head and face pain experienced by the first patient. ${ }^{10}$ ICA is usually unilateral but can be bilateral in up to $10 \%$ of patients. ${ }^{9}$ Compared to unilateral disease, bilateral disease causes a greater obstruction of cerebral blood flow and is more likely to lead to cerebral ischaemia. A concurrent vertebral artery dissection can also be found in more than onefifth of cases."

ICA dissection can occur spontaneously or can be attributed to trauma in $40 \%$ of cases. ${ }^{1,3,12}$ The mechanism of injury is usually blunt force trauma, such as a direct blow to the neck or sudden neck movements. ${ }^{13}$ Other risk factors for ICA dissection include systemic conditions and congenital tissue defects: fibromuscular dysplasia, Marfan's syndrome, Ehlers-Danlos syndrome, a-1 antitrypsin deficiency, Behçet's syndrome, and 
osteogenesis imperfecta Type 1.,3,12 The disease is caused by a tear in the wall of the vessel creating a false lumen and the formation of a mural haematoma, thereby compressing and narrowing the true lumen. ${ }^{3}$ Formation of a haematoma leads to mass effect on the surrounding structures, and in the case of a cervical ICA dissection, it impacts on the other structures within the carotid sheath. ${ }^{14}$

It has been suggested in the literature that ICA dissection should be diagnosed through duplex ultrasonography or MRI. ${ }^{3,14}$ A further CT angiogram can then help localise and evaluate the extent of the ICA dissection. 2,14 In the case of a haematoma, MRI scans show hyperintensity on $\mathrm{T} 1$ and $\mathrm{T} 2$ images or a delineation around the blood clot. $^{2}$ One of the limitations of duplex ultrasonography is that unless there is a change in the blood flow caused by the dissection, the diagnosis can be missed..$^{15}$ In the authors local practice, patients presenting with a vocal cord palsy are initially referred for a CT scan encompassing the skull base to the diaphragm. In both cases, contrast CT scans using split-bolus CT provided good evidence of ICA dissection. MRI was used in one case to confirm the diagnosis. This was supported by the findings of one study that showed that the evaluation accuracy of $\mathrm{CT}$ and MRI are comparable in the diagnosis of carotid and vertebral artery dissection. ${ }^{16}$
The prognosis of ICA dissection is generally good and there is full or almost full recovery of symptoms in up to $80 \%$ of cases. ${ }^{7}$ Although it can resolve spontaneously in a large number of patients, ICA dissection is usually managed with antithrombotics to reduce the risk of stroke, usually antiplatelets or warfarin;1,6,17 however, there is no compelling evidence to favour one over the other. ${ }^{18}$ Although it is does not treat the dissection itself, the antithrombotic therapy leads to a higher rate of recanalisation within the first 2-3 months. ${ }^{19}$ With the right management, the outcome of an ICA dissection is good.

\section{CONCLUSION}

After excluding malignancy in a patient with vocal cord palsy, particularly in a sudden onset or if associated with facial or neck pain or headache, physicians should consider ICA dissection as another potential cause. Although rare, ICA dissection can lead to serious complications, such as embolic stroke, transient ischaemic attack, or aneurysms.,20,21 MRI scans have been suggested as the radiological investigation of choice, however, in both cases, split-bolus CT has shown to provide good evidence of ICA dissection. Prompt and appropriate treatment should be started using antithrombotics and, in most cases, this will lead to a good outcome.

\section{References}

1. Popov P et al. Vocal cord paralysis as the first sign of spontaneous carotid dissection in a patient with extracranial internal carotid artery aneurysm. Vasc Endovascular Surg. 2016;50(1):52-6

2. Houser OW et al. Spontaneous cervical cephalic arterial dissection and its residuum: angiographic spectrum. Am J Neuroradiol. 1984;5(1):27-34.

3. Wessels $\mathrm{T}$ et al. Vocal cord palsy resulting from spontaneous carotid dissection. Laryngoscope. 2003;113(3):537-40.

4. Hou J. Fetal warfarin syndrome. Chang Gung Med J. 2004;27(9):691-5.

5. Debette $\mathrm{S}$ et al. Epidemiology, pathophysiology, diagnosis, and management of intracranial artery dissection. Lancet Neurol. 2015:14(6):640-54.
6. Larsson SC et al. Prognosis of carotid dissecting aneurysms: results from CADISS and a systematic review. Neurology. 2017;88(7):646-52.

7. Fusco MR, Harrigan MR. Cerebrovascular dissections a review part I: spontaneous dissections. Neurosurgery. 2011;68(1):242-57.

8. Majeed A et al. A rare presentation of spontaneous internal carotid artery dissection with Horner's syndrome, VIIth, Xth and XIIth nerve palsies. Oxford Med Case Reports. 2016;(10):255-8.

9. Ishigami D, Ota T. Spontaneous bilateral cervical internal carotid artery dissection treated with simultaneous bilateral carotid artery stenting: a case report. NMC Case Rep J. 2019;6(3):71-4.

10. Kessler LA, Abla A. Syndrome of the cervical plexus caused by high cervical nerve root compression.
Neurosurgery. 1991;28(4):506-9.

11. Van Den Berg JSP et al. The role of Type III collagen in spontaneous cervical arterial dissections. Ann Neurol. 1998;43(4):494-8

12. Olin JW et al. State of the science and critical unanswered questions: a scientific statement from the American Heart Association. Circulation. 2014;129(9):1048-78.

13. Galyfos $\mathrm{G}$ et al. Traumatic carotid artery dissection: a different entity without specific guidelines. Vasc Spec Int. 2016;32(1):1-5.

14. Nguyen TTJ et al. Vocal cord paralysis secondary to spontaneous internal carotid dissection: case report and systematic review of the literature. J Otolaryngol Head Neck Surg. 2013;42(1):34.

15. Spitzer $\mathrm{C}$ et al. Isolated hypoglossal nerve palsy caused by carotid artery dissection - the necessity 
of MRI for diagnosis. J Neurol. 2001;248(10):909-10.

16. Provenzale JM, Sarikaya B. Comparison of test performance characteristics of MRI, MR angiography, and CT angiography in the diagnosis of carotid and vertebral artery dissection: a review of the medical literature. AJR Am J Roentgenol. 2009;193(4):1167-74.

17. Baracchini $\mathrm{C}$ et al. Neurosonographic monitoring of 105 spontaneous cervical artery dissections: a prospective study. Neurology. 2010;75(21):1864-70.

18. Markus HS et al. Antiplatelet treatment compared with anticoagulation treatment for cervical artery dissection (CADISS): a randomised trial. Lancet Neurol. 2015:14(4):361-7.

19. Schievink WI. The treatment of spontaneous carotid and vertebral artery dissections. Curr Opin Cardiol. 2000;15(5):316-21.

20. Bogousslavsky J, Regli F. Ischemic stroke in adults younger than 30 years of age: cause and prognosis. Arch Neurol. 1987;44(5):479-82.

21. Goodman JM et al. Hemilingual paralysis caused by spontaneous carotid artery dissection. Arch Neurol. 1983;40(10):653-4 